\title{
Ultra-trace measurement of Dechloranes to investigate food as a route of human exposure
}

\author{
Benjamin L'Homme ${ }^{\mathrm{a}, 1}$, Chiara Calaprice ${ }^{\mathrm{a}, \mathrm{b}, \mathrm{c}, 1}$, Cosima Damiana Calvano ${ }^{\mathrm{c}}$, Carlo Zambonin ${ }^{\mathrm{c}, \mathrm{d}}$, \\ Riccardo Leardi ${ }^{\mathrm{e}}$, Jean-François Focant ${ }^{\mathrm{a}, *}$ \\ ${ }^{a}$ CART, Organic and Biological Analytical Chemistry Group, Chemistry Department, University of Liège, Allée de la Chimie 3, B6c Sart-Tilman, B-4000 Liège, Belgium \\ ${ }^{\mathrm{b}}$ Dipartimento di Ingegneria Civile, Ambientale, del Territorio, Edile e di Chimica, Politecnico di Bari, Via Orabona, 4, 70125 Bari, Italy \\ 'Dipartimento di Chimica, Università degli Studi di Bari “Aldo Moro", via Orabona 4, 70125 Bari, Italy \\ 'Centro Interdipartimentale di Ricerca S.M.A.R.T., Università degli Studi di Bari “Aldo Moro", c/o Dipartimento di Chimica, via Orabona 4,70125 Bari, Italy \\ e Department of Pharmacy, Unit of Pharmaceutical and Food Chemistry and Technology, Via Brigata Salerno (ponte), University of Genoa, I-16147 Genoa, Italy
}

\section{H I G H L I G H T S}

- This is the first report on levels of Dechloranes in European Food and feed.

- A specific GC-QQQMS/MS method was developed.

- Most selected Dechloranes were detected in European food and feed.

- Dechloranes are at the $\mathrm{pg} / \mathrm{g}$ fat levels in food.

- A Dechlorane dietary intake was estimated for the Belgian population.

\section{A R T I C L E I N F O}

\section{Article history:}

Received 11 May 2015

Received in revised form 9 July 2015

Accepted 16 July 2015

Available online 24 August 2015

\section{Keywords:}

Dechloranes

Ultra-trace measurement

Experimental design

Food analysis

Human intake

Triple quadrupole tandem mass

spectrometry

\begin{abstract}
A B S T R A C T
Dechloranes, including Dechlorane Plus (syn- and anti-isomers), Dechlorane 602, Dechlorane 603, Dechlorane 604, Chlordene Plus, and Mirex are used as flame-retardants and were recently found in human serum of the European population. In order to investigate if food consumption would possibly be a significant route of exposure, we developed a method for the measurement of Dechloranes in food and feed. We showed that it was possible to extend the scope of the regular polychlorinated dibenzo-p-dioxins (PCDDs), polychlorinated dibenzofurans (PCDFs), dioxin like (DL-), and non-dioxin like (NDL-) regulated PCBs clean-up and fractionation procedure to Dechloranes and that no compound degradation occurred during the strong acidic treatments used for lipid digestion. Dechloranes were measured by gas chromatography coupled to triple quadrupole mass spectrometry (GC-QQQMS/MS). We optimized injection parameters by face centered experimental design (FCD). The electron ionization fragmentation was investigated to set appropriate multiple reaction monitoring (MRM) transitions. Instrumental and method limits of quantitation (iLOQs and mLOQs) were determined following EU guidelines for dioxin analyses in food. A total of 88 samples were analyzed to assess the prevalence of this route of exposure to humans. Average levels of the sum of Dechloranes ranged from 10 to $31 \mathrm{pg} / \mathrm{g}$ fat, with the exception of fish, feed additives, and corn that were reported in $\mathrm{pg} / \mathrm{g}$ wet weight at average levels of 9, 12, and $2 \mathrm{pg} / \mathrm{g}$ ww. Based on Belgian food habits, a dietary intake was estimated to be $136 \mathrm{pg} /$ day. The relatively low reported levels indicate that other routes of human exposure should be considered.
\end{abstract}

(c) 2015 Elsevier Ltd. All rights reserved.

\section{Introduction}

Dechloranes are emerging persistent organic pollutants (POPs) issued from a family containing structural analogue compounds,

\footnotetext{
* Corresponding author.

E-mail address: JF.Focant@ulg.ac.be (J.-F. Focant).

1 These authors contributed equally to this work.
}

namely Mirex $\left(\mathrm{C}_{10} \mathrm{Cl}_{12}\right)$, Dechlorane Plus (DP, $\mathrm{C}_{18} \mathrm{H}_{12} \mathrm{Cl}_{12}$ ), Dechlorane 602 (Dec 602, $\mathrm{C}_{14} \mathrm{H}_{4} \mathrm{Cl}_{12} \mathrm{O}$ ), Dechlorane 603 (Dec 603, $\mathrm{C}_{17} \mathrm{H}_{8} \mathrm{Cl}_{12}$ ), Dechlorane 604 (Dec 604, $\mathrm{C}_{13} \mathrm{H}_{4} \mathrm{Br}_{4} \mathrm{Cl}_{6}$ ), and Chlordene Plus ( $\left.\mathrm{CP}, \mathrm{C}_{15} \mathrm{H}_{6} \mathrm{Cl}_{12}\right)$. They are norbornene derivatives that exhibit flame-retardant and pesticide properties and are used as replacement of regulated compounds such as Mirex (also called Dechlorane) (INCHEM, 1984) and decabromodiphenyl ether (deca-BDE, BDE-209) (Pakalin et al., 2007). DP and other Dechloranes have 
been extensively used as additive in various synthetic products such as nylon or plastic like polypropylene (OxyChem, 2007) and have recently been reported at low levels in dust collected from various environments (Cao et al., 2014; Dodson et al., 2012). In addition, some break down and dechlorinated products of DP (Muñoz-Arnanz et al., 2012), of Dec 602 (Shen et al., 2012), and mixed halogenated analogues of Dec 604 (Jobst et al., 2013) have also been found in the environment. Besides environmental issues, humans are exposed to these chemicals and, so far, barely any data about toxicity, metabolization, and prevalence in human are available. Nevertheless, very recent human biomonitoring studies have reported levels at the $\mathrm{ng} / \mathrm{g}$ lipid in human serum from Norway (Cequier et al., 2013) and France (Brasseur et al., 2014), as well as in breast milk from Canada (Zhou et al., 2014).

For the present study, we investigated food consumption as a possible route of human exposure to Dechloranes in Belgium. Such an exercise has only been carried out recently in Korea (Kim et al., 2014), and in Japan (Kakimoto et al., 2014). Both studies were using gas chromatography (GC) coupled to high-resolution mass spectrometry (HRMS) sector instruments for measurements at the $\mathrm{pg} / \mathrm{g}$ level. As far as we know, no data are currently available regarding Dechlorane exposure from food consumption in Europe, where no Dechlorane production sources have been identified so far, despite the fact that $\mathrm{ng} / \mathrm{g}$ lipid levels have been reported in humans (Brasseur et al., 2014; Cequier et al., 2013). Because of the emerging character of these analytes, the first part of the study has been dedicated to the development of a specific method for the analysis of 6 Dechloranes: Mirex, Dec 602, Dec 603, CP, DP syn, and DP anti isomers. We based our analytical approach on one of our recent report on the full validation of a GC isotope dilution (ID) triple quadrupole tandem in space mass spectrometry (QQQMS/MS) method for the measurement of dioxins in food and feed at the low pg level under the European Legislation (L'Homme et al., 2015). To optimize the setup of the Dechlorane dedicated ultra-trace measurement method, we investigated large volume injection (LVI) by using a programmed temperature vaporizing (PTV) injector operated in solvent vent mode. Full factorial design (FFD) and face-centered design (FCD) were used to select optimum inlet parameters. Most efforts were focused on the three most relevant factors such as vent flow, vent pressure, and vent temperature but also on other minor injection parameter, such as purge flow, in order to maximize method sensitivity. On the MS side, specific multiple reaction monitoring (MRM) transitions were selected from typical Dechlorane fragmentation patterns as they can fragment following a retro Diels-Alder reaction of the norbornene moiety of the molecule (Brasseur et al., 2012; Shen et al., 2012). In this paper, we report on the development of the analytical method and its use for the measurement of Dechloranes in selected food and feed samples. It was applied to 88 samples to produce a first estimate of Dechlorane dietary intake for the Belgian population.

\section{Experimental}

\subsection{Chemicals and consumables}

Solvents (hexane, toluene, methanol, ethanol and dichloromethane) were Picograde ${ }^{\circledR}$ reagents (LGC Promochem, Wesel, Germany). Nonane puriss analytical-reagent grade standard for GC was purchased from Fluka (Steinheim, Germany). Water was obtained from a Milli-Q Ultrapure water purification system (Millipore, Brussels, Belgium). Solvent batches were tested for contamination before use. Disposable PTFE columns for the automated clean-up were obtained from Fluid Management Systems (FMS Inc., Waltham, MA, USA). Chromatographic pure grade helium gas, 99.9999\% alphagaz 2 was purchased from Airliquide (Paris, France). Technical N27 grade liquid $\mathrm{CO}_{2}$ was used for PTV cooling (Airliquide, Paris, France). Sodium sulfate and diatomaceous earth were purchased from VWR International (Radnor, PA, USA). Standards of DP syn, DP anti, as well as ${ }^{13} \mathrm{C}_{10}$-labeled internal standards DP syn and ${ }^{13} C_{10}$-labeled internal standard Dec 602 were supplied by Cambridge Isotope Laboratories (CIL, Andover, MS, USA). CP standard was bought from Wellington Laboratories (Guelph, ON, Canada). Mirex standard was purchased from Cluzeau Info Labo (France). Dec 602, Dec 603 and Dec 604 standards were purchased from Toronto Research Chemical Inc. (Toronto, ON, Canada). The quantitation of DP isomers was performed using ${ }^{13} \mathrm{C}_{10}$-labeled DP syn internal standard, whereas mirex, Dec 602, 603, 604 and CP were quantitated against ${ }^{13} \mathrm{C}_{10}$-labeled Dec 602 internal standard. The EC-1414 solution of ${ }^{13} \mathrm{C}_{12}$-labeled PCB-80, from CIL, was used as recovery standard. This standard was used to assess the efficiency and the loss of compounds during the sample preparation (internal standard vs recovery standard). The quantitation was however not affected by any loss of compounds since all analytes were quantitated by isotopic dilution (ID) (analyte vs internal standard) during which process analytes and internal standards were lost in the same proportions. Calibration curves were prepared from intermediate stock solutions at $1 \mathrm{ng} / \mu \mathrm{L}$ and consisted in 57 calibration points (Supplementary Information, Table S1).

\subsection{Samples}

A total of 88 samples were collected for analyses. Sample matrices consisted in milk, chicken, pork, eggs, pure animal fat, vegetable oil, salmon, feed additives, and corn. A group of 77 samples of different food and feed matrices were randomly selected from samples entering our ISO17025 accredited routine dioxin laboratory under the EU monitoring program. The remaining 11 samples ( 5 salmons and 6 chickens) were collected from regular shops and supermarkets in the area of Liege, Belgium, in January 2015 , to complete the missing matrices. Samples were frozen and stored until use. During the study, 16 procedural blank samples were also analyzed.

\subsection{Sample preparation: extraction and clean up}

All samples were prepared in a similar way than for polychlorodibenzo-p-dioxin (PCDD), polychloro-dibenzofuran (PCDF), and polychlorobiphenyl (PCB) analysis in an ISO 17025 environment. Details on the method are available in previous reports (Focant et al., 2006, 2001). Briefly, for all the matrices fat extraction was required because of the lipophilicity of Dechloranes and it was performed using accelerated solvent extraction (ASE ${ }^{\mathrm{TM}}$ 350, Dionex, Thermo Fisher Scientific). Labeled internal standard spike was carried out before fat extraction for matrices such as salmon and feed, and directly on the extracted fat for the other matrices. After this step, samples underwent a manual acidic silica column pre-clean up and then the automated PowerPrep ${ }^{\mathrm{TM}}$ system (FMS Inc, Waltham, USA) was used for deep clean-up and compounds fractionation (Focant et al., 2004). Samples were loaded on a multi-layer acid/basic/neutral (ABN) silica column for lipid breakdown, then passed through a partly deactivated basic alumina column for interference removal and compound fractionation, and finally ended up in a carbon-based column for separation of non-planar from planar species. For PCDD/F and PCB classical clean-up, two fractions were collected and sent to instrumental analysis. Dechloranes were collected with non-planar species (mono-ortho (MO-)PCBs and indicator (I-)PCBs) by forward elution with a mixture of hexane/dichloromethane 50:50. This fraction was evaporated in a dedicated tube using a sensor-equipped TurboVap II Workstation 
(Caliper Life Science, Teraflene, Belgium), then transferred in GC vials containing nonane $(90 \mu \mathrm{L})$ as a keeper. Solvent exchange to nonane was carried out using a RapidVap (Labconco, Kansas City, MO, USA). The ${ }^{13} \mathrm{C}_{12}$-labeled PCB-80 recovery standard was added prior instrumental analysis. Details on the full procedure for PCDD/Fs and PCBs can be found elsewhere (Focant et al., 2001; Pirard et al., 2002). Blanks were prepared following the same procedure as for their corresponding real samples and consisted in a mixture of sodium sulfate (washed with hexane) and diatomaceous earth (after washing with methanol and dichloromethane) for all the matrices, except for pure fat and milk, for which hexane and water were used, respectively.

\subsection{GC-MS/MS conditions}

For instrumental analysis, we used a $7000 \mathrm{C}$ gas chromatography triple quadrupole mass spectrometer (GC-QQQMS/MS) from Agilent (Palo Alto, CA, USA) equipped with a 7890B GC oven, a programmable temperature vaporization (PTV) inlet, and a 7693A automated liquid sampler (ALS). We injected $5 \mu \mathrm{L}$ of the final purified extract into the PTV inlet operating in solvent vent mode and cooled by liquid $\mathrm{CO}_{2}$. The inlet temperature program and solvent vent parameters were optimized by means of experimental design and were: inlet initial temperature of $45^{\circ} \mathrm{C}$ for $1.3 \mathrm{~min}$, then ramped up at $720^{\circ} \mathrm{C} / \mathrm{min}$ to $320^{\circ} \mathrm{C}$; vent flow was $120 \mathrm{~mL} / \mathrm{min}$ at vent pressure of 10.5 psi. Purge flow was set to $1200 \mathrm{~mL} / \mathrm{min}$ after $5 \mathrm{~min}$. All the computations and graphs were performed using multiple linear regression routines written in Matlab (Mathworks Inc., Natrick, USA) already used in a previous study (Mac Namara et al., 2005). The GC column was an Agilent DB-5 ms ultra inert $(60 \mathrm{~m} \times 0.25 \mathrm{~mm} \times 0.25 \mu \mathrm{m})$, the same used in routine for PCDD/F, DL- and NDL-PCB analysis, allowing to analyse Dechloranes during regular controls for those compounds without changing the analytical setup. The GC oven temperature program was adapted due to the high molecular weight of Dechloranes with a sharper ramp to elute Dechloranes faster: start at $140{ }^{\circ} \mathrm{C}$ for $2.6 \mathrm{~min}$, ramp at $100{ }^{\circ} \mathrm{C} / \mathrm{min}$ to $320^{\circ} \mathrm{C}$ and hold for $21.1 \mathrm{~min}$ (total run time of $25.5 \mathrm{~min}$ ). The transfer line temperature was held at $320^{\circ} \mathrm{C}$. On the MS side, we used the electron ionization (EI) at $70 \mathrm{eV}$ energy and temperature of $280^{\circ} \mathrm{C}$. Temperature of the quadrupoles was set at $150{ }^{\circ} \mathrm{C}$ and multiple reaction monitoring (MRM) transitions were recorded at 'wide' mass resolution, which means 1.2 Da on the Agilent software. Ultrapure Nitrogen at $1.5 \mathrm{~mL} / \mathrm{min}$ and Helium at $2.25 \mathrm{~mL} / \mathrm{min}$ were used respectively as collision gas and quench gas in the collision cell. Dwell times were selected during method optimization to increase the sensitivity as much as possible and to adjust the acquisition frequency to get ten data points per peak. Calibration and autotune were performed in the EI high sensitivity mode. Retention time locking was performed with PCB-105 allowing change and reinstallation of the column while keeping reproducible retention times. Mass Hunter version B.07.00 was used for acquisition and quantitative analysis.

\section{Results and discussion}

\subsection{Sample clean-up}

The study aimed at developing a multi-analyte procedure for selected persistent organic pollutant (POP) analysis. We therefore started by investigating and optimizing the sample preparation steps. From previous experience, we knew that typical dioxin sample preparation procedures were usable for Dechlorane measurements in serum (Brasseur et al., 2014). Because of the high lipid content of some food matrices, we had to use acidic silica to chemically degrade the fat prior fractionation. A manual high capacity (up to $5 \mathrm{~g}$ of fat) acidic silica column treatment was implemented in combination with the automated system involving mixed bed acid/basic silica, alumina and carbon columns. We performed recovery experiments across each clean-up step to investigate the possible degradation of Dechlorane compounds under such acidic treatment. We saw that all Dechloranes strongly resist (all recovery rates close to $100 \%$ ) to repeated acidic treatment. In Fig. 1, we report the detailed fractionation study where we measured Dechlorane levels in all fractions produced by the automated system performing in the dioxin configuration. It appeared that all Dechlorane compounds were present in the MO-PCBs fraction (non planar species). This demonstrated that the classic dioxin clean up and fractionation procedure we reported earlier (Focant et al., 2001) can be used for the sample preparation in Dechlorane analyses.

\subsection{Experimental design for optimization of PTV parameters}

\subsubsection{Structure of the experimental design}

The programmed temperature vaporizing (PTV) inlet was operated in solvent vent mode. Inlet parameters such as initial inlet temperature (T), vent flow (VF), and vent pressure (VP) were settled by means of experimental design to ensure method sensitivity. Since we had no preliminary data about Dechlorane background levels in food and feed matrices, we had to work on a wide dynamic range, with special focus on ultra-trace levels. As the first interface affecting sensitivity, we optimized inlet parameters by maximizing peak area while taking into account the quality of the data through peak shape (neither fronting nor tailing). A preliminary $2^{3}$ full factorial design (FFD) was performed to explore the experimental domain and to assess a suitable model to postulate for a further more rigorous experimental design investigation. Thereafter a face-centered design (FCD) on three factors was adopted to take into account quadratic effects and interactions between variables (Supplementary Information, Table S2). Central point and boundaries were selected according to the screening results of the FFD. The ranges in which inlet temperature (T), vent flow (VF), and vent pressure (VP) were evaluated were $30-60^{\circ} \mathrm{C}$, $20-100 \mathrm{~mL} / \mathrm{min}$, and $1-20 \mathrm{psi}$, respectively. The FCD allowed the estimation of the coefficients (effects) in the postulated model (Eq. (1)):

$$
\begin{aligned}
y= & b_{0}+b_{1} x_{1}+b_{2} x_{2}+b_{3} x_{3}+b_{12} x_{12}+b_{13} x_{13}+b_{23} x_{23}+b_{11} x_{1}^{2} \\
& +b_{22} x_{2}^{2}+b_{33} x_{3}^{2}
\end{aligned}
$$

where

$-x_{i}$ is the experimental factor representing initial inlet temperature $(\mathrm{T})(i=1)$, solvent vent flow (VF) $(i=2)$ and solvent vent pressure (VP) $(i=3)$;

$-x_{i j}$ is the factor representing the interaction between factor $i$ and $j$, or the quadratic factor if $i=j$;

$-b_{i}$ is the effect of the factor $i$;

$-b_{i j}$ is the effect of the interaction between factors $i$ and $j$, or the quadratic effect for $i=j$;

$-b_{0}$ is the response in the center of the domain, where all the factor are set equals to 0 .

For each of the Dechloranes, two responses were recorded in MRM mode: peak area $\left(y_{1}\right)$, and peak symmetry $\left(y_{2}\right)$. Both responses were calculated in the quantitative analysis software. A total of 17 experiments were performed with three replicates in the center. They were conducted in random order using a $10 \mathrm{pg} / \mu \mathrm{L}$ mixture of each of the Dechlorane standards in nonane. 


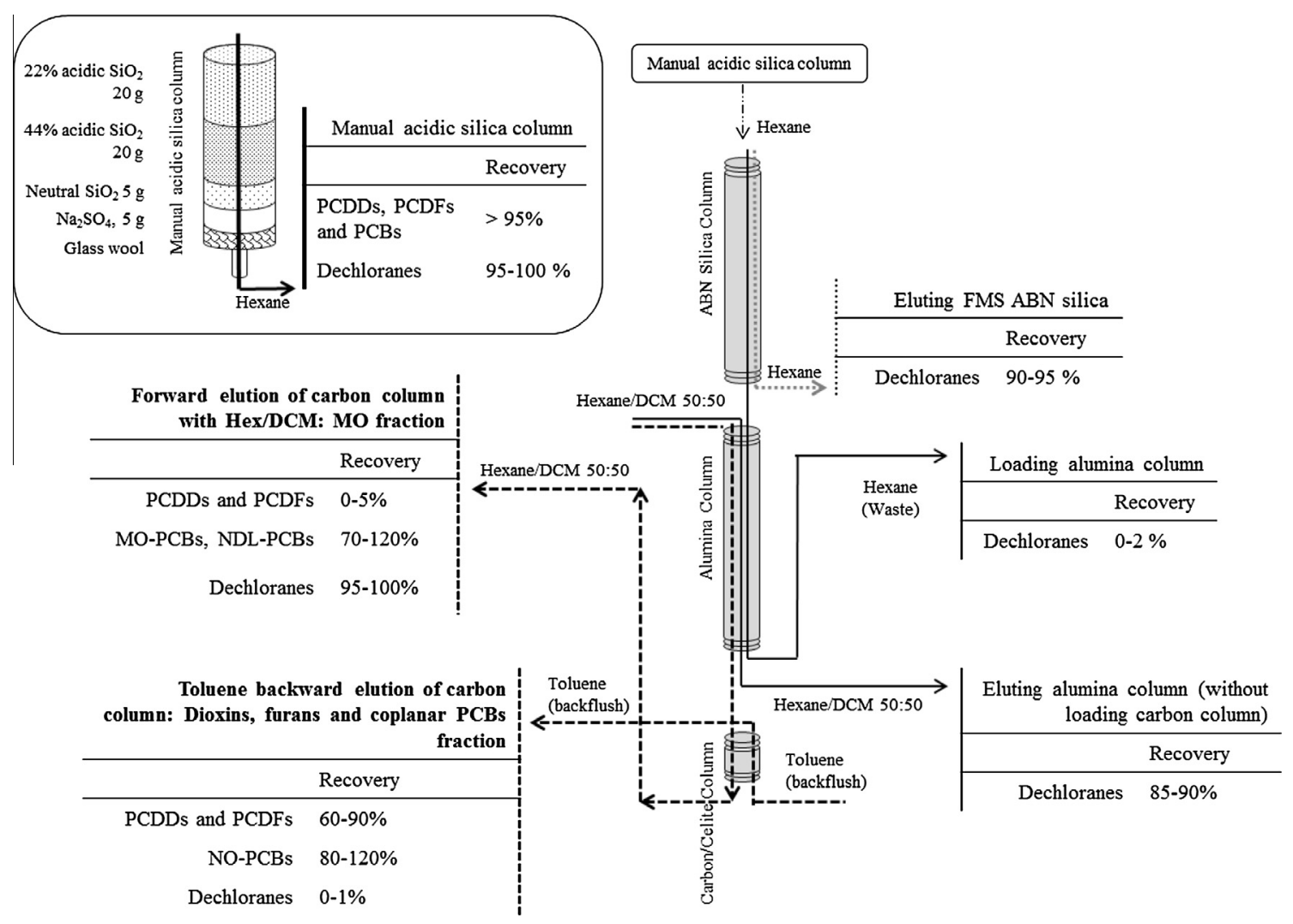

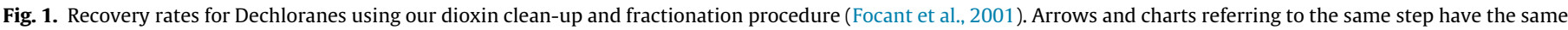

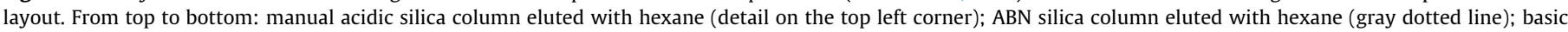

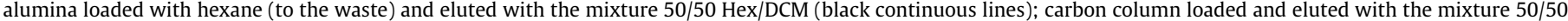

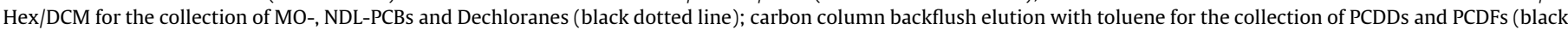
dotted line).

\subsubsection{Peak area and peak symmetry}

Significant effects for peak area $\left(y_{1}\right)$ were highlighted in the model for the target compounds (Supplementary Information, Fig. S1). At this concentration $(10 \mathrm{pg} / \mu \mathrm{L})$, Dec 604 was not detectable and was therefore not represented. Significant coefficients were almost the same for all compounds, and were mostly related to temperature ( $b_{1}$ and $b_{11}$ ), which had a negative quadratic effect on peak area, and to vent flow $\left(b_{2}\right)$, which had a positive linear effect. Vent pressure $\left(b_{3}\right)$ was not statistically significant, but was close to have a significant negative linear effect for Mirex and DP syn. For all the models no lack of fit was observed, since variance of residuals was comparable to the variance of replicates.

Peak symmetry was calculated at $10 \%$ height of the peak as the ratio between the distance from the tail part of the peak to the center of the apex, and from the front part of the peak to the center of the apex. A symmetry value of 1.0 meant that the peak was balanced, whereas values lower and higher than 1 indicated respectively peak fronting and tailing. In this work peak symmetry values between 0.9 and 1.1 were considered as acceptable, although the best symmetry was reached at 1.0 , because peak symmetry was calculated on raw experimental data without smoothing. We therefore expected experimental variation on the peak apex when the true center of the peak did not correspond to the highest point. We observed that temperature, vent flow and their interaction had significant effects for peak symmetry $\left(y_{2}\right)$ for some analytes $\left(b_{1}, b_{2}, b_{12} \neq 0\right)$, even if this response was effected by high experimental variability (Supplementary Information, Fig. S2). We also noted that vent pressure $\left(b_{3}\right)$ was almost significant (positive effect) at $95 \%$ confidence for Mirex only.

\subsubsection{Optimum parameters}

We saw that peak area dramatically decreased with temperature above and below $45{ }^{\circ} \mathrm{C}$ because of its strong negative quadratic effect, and mostly increased with vent flow. In addition, vent flow had a positive significant effect also on peak symmetry. Vent pressure, even if not statistically significant for both responses, showed an overall negative effect on peak area and rather positive effect on peak symmetry. The optimum values of the three factors consisted in the compromise between maximum peak area and symmetric peak shape (peak symmetry between 0.9 and 1.1). For this reason, even if the optimum vent pressure to maximize peak area appeared to be $1 \mathrm{psi}$, this value led to peak fronting (peak symmetry <1) as shown in the left part of Fig. 2, that shows peak area response surfaces (top) and overlapped isoresponse curves for peak area and peak symmetry (bottom) of Mirex as an example, at the two different vent pressures that led to the highest peak area. Therefore, the most profitable combination between the two responses was: VP at $10.5 \mathrm{psi}$ (intermediate level in the domain), VF at $100 \mathrm{~mL} / \mathrm{min}$ (highest level in the domain) and temperature at $45^{\circ} \mathrm{C}$ (intermediate level in the domain), as shown in the overlapped isoresponse plots on the bottom right of Fig. 2.

To go further, from the peak area response curves of Fig. 2, we saw that the peak area response might increase even more outside the experimental domain with a higher vent flow, while still keeping acceptable symmetry at 10.5 psi. For this reason, three replicates at a vent pressure of $120 \mathrm{~mL} / \mathrm{min}$ (outside the experimental domain), temperature at $45^{\circ} \mathrm{C}$ and pressure at $10.5 \mathrm{psi}$ were recorded to check whether or not the response increased significantly in the expected direction. Indeed, the peak area in these con- 

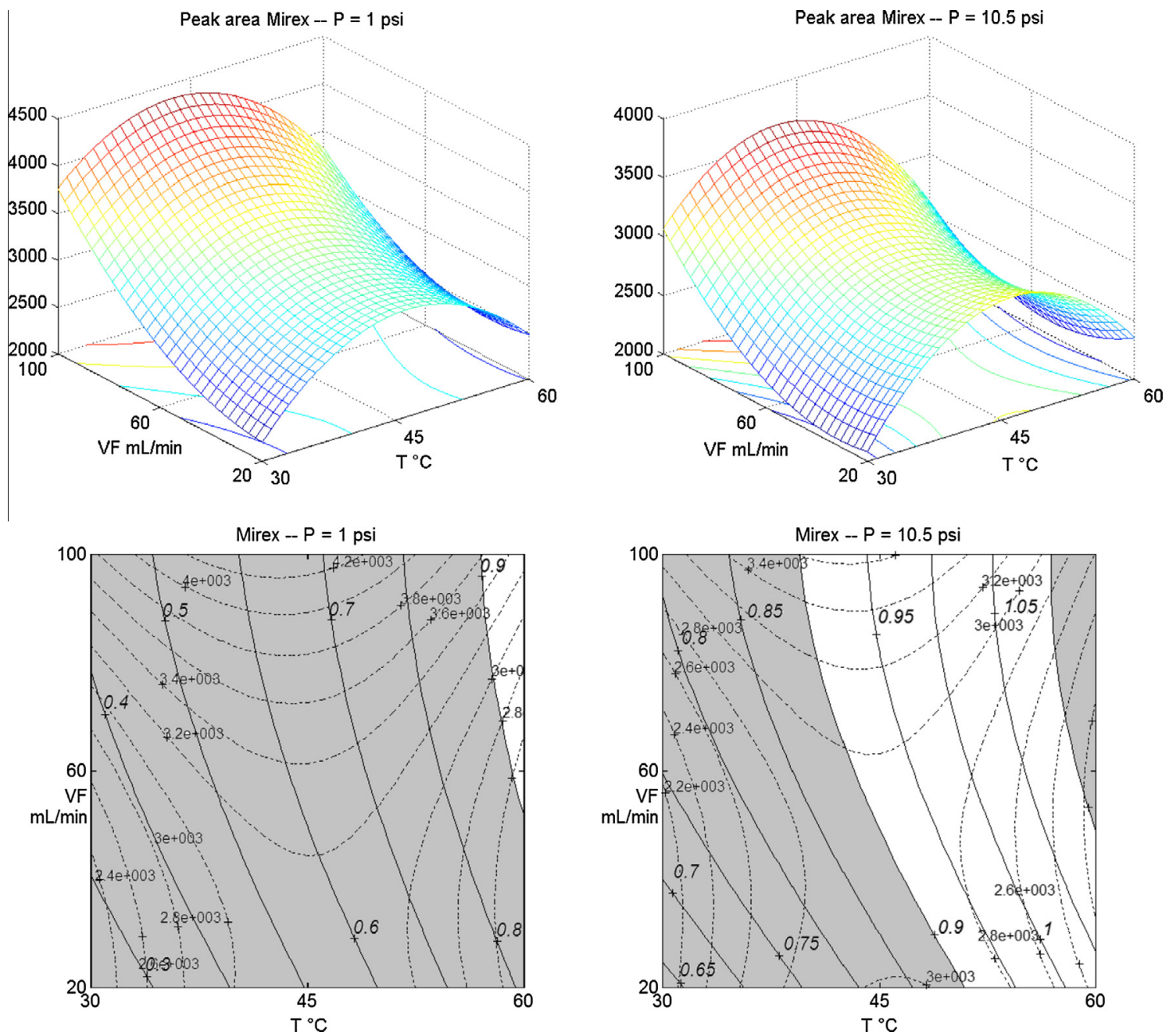

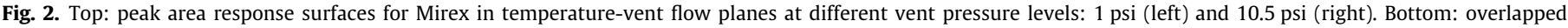

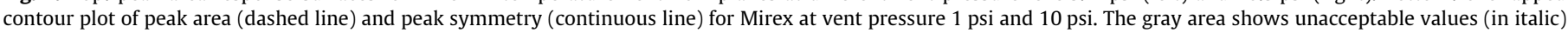
for peak symmetry in the experimental domain (outside the 0.9-1.1 range).

ditions was statistically higher (using $t$-test) than at VF of $100 \mathrm{~mL} / \mathrm{min}$. At this point, even without a full optimization but still with enough improvements using the experimental design for Dechloranes analysis, the best injection conditions were settled as VF at $120 \mathrm{~mL} / \mathrm{min}$, T at $45^{\circ} \mathrm{C}$, and VP at $10.5 \mathrm{psi}$. Moreover, to deeper validate the experimental design, we performed reproducibility experiments for peak area to highlight memory effect (carry over) in the inlet, resulting in a constant increase of the area of the peaks. Increasing the purge flow from $100 \mathrm{~mL} / \mathrm{min}$ to $1200 \mathrm{~mL} / \mathrm{min}$ was necessary to properly free the inlet from any residual Dechloranes when its initial temperature (temperature also kept when the system is on standby) was set at $45^{\circ} \mathrm{C}$, a temperature significantly lower than the boiling point of the compounds.

\subsection{Triple quadrupole mass spectrometry operating in tandem mode}

Major parameters of the mass spectrometer were identical than for dioxin analysis as already described in a previous validation study (L'Homme et al., 2015). A specific optimization was however necessary and consisted in determining the appropriate MRM transitions by a set of experiments. We first collected a full mass spectrum of every compound by only scanning on the first quadrupole (MS1 scan). The second experiment consisted in selecting the base peak as precursor and colliding it in the collision cell at different energies (from 5 to $40 \mathrm{eV}$ ). We scanned on the third quadrupole (MS2 scan) to collect a full spectrum from the precursor. We finally selected the base peak coming from the fragmentation of the precursor to set the MRM transition. We optimized the collision energy to obtain the highest signal. However, changing the latter parameter did not significantly influence the fragmentation pattern and the relative intensities of all fragments in the working range. It rather changed the overall intensity of the mass spectrum.

The routes of fragmentation are illustrated in Fig. 3. For DP, CP, and Dec 602, the retro Diels-Alder product (the hexachlorocyclopentadiene ion - HCCPD) was the base peak obtained after the electro ionization (EI). It was especially expected for Dec 602 as a furan molecule (aromatic) was also produced, which was a strong driving force for the reaction. After fragmentation, the HCCPD lost one chlorine atom, hence the MRM transition for those compounds: $m / z 272>m / z 237$ (25 eV collision energy (CE)). Mirex also fragmented according to this transition even though not strictly speaking coming from a retro Diels-Alder process since the molecule does not have a double bond. For Dec 603, we observed the HCCPD ion coming from retro Diels-Alder reaction but also a significant fragment at $m / z 262.8$, likely coming from the fragmentation in the middle ring with the concomitant loss of $\mathrm{Cl}$. We chose the latter precursor ion because of interferences preventing proper quantitation with the $\mathrm{m} / \mathrm{z} 272$ precursor. The product ion subse- 


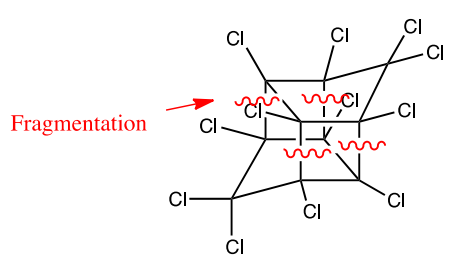

Mirex<smiles>ClC1=C(Cl)C2(Cl)C3CCC4C(CCC3C1(Cl)C2(Cl)Cl)C1(Cl)C(Cl)=C(Cl)C4(Cl)C1(Cl)Cl</smiles>

DP<smiles>ClC1=C(Cl)C2(Cl)C3C(CC4C(Cl)(Cl)C5(Cl)C4(Cl)C(Cl)=C(Cl)C35Cl)C1(Cl)C2(Cl)Cl</smiles>

CP<smiles>ClC1=C(Cl)C2(Cl)C3C1C1OC3C3(Cl)C(Cl)=C(Cl)C2(Cl)C1(Cl)C3(Cl)Cl</smiles>

Dec 602

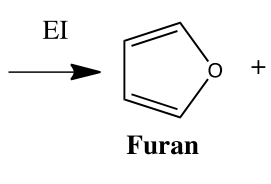

Retro Diels-Alder product

Hexachlorocyclopentadiene

$$
\text { HCCPD }(\mathrm{m} / \mathrm{z}=272)
$$

Q1

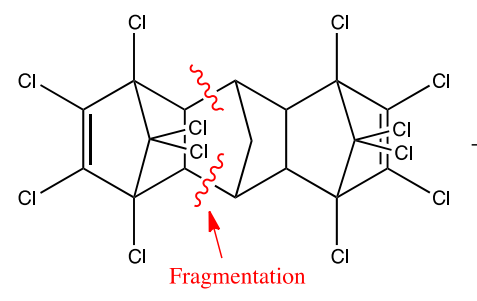

Dec 603

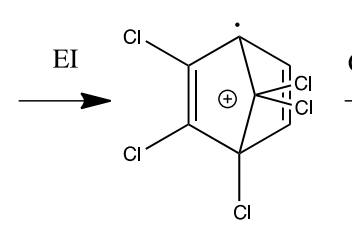

No retro Diels-Alder product $(\mathrm{m} / \mathrm{z}=262.8)$
$\mathrm{Q1}$<smiles>CC(C)(C)OOC(C)(C)C</smiles>

$$
\begin{gathered}
\text { Precursor - Cl } \\
\begin{array}{c}
(\mathrm{m} / \mathrm{z}=227.8) \\
\text { Q3 }
\end{array}
\end{gathered}
$$

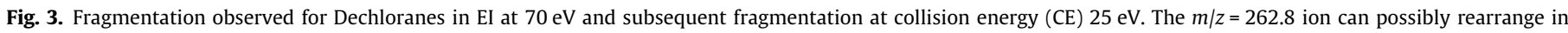
tropylium ion.

quently consisted in the loss of one chlorine atom, hence the transition for Dec 603: $\mathrm{m} / z 262.8>\mathrm{m} / z 227.8$ (25 eV CE).

\subsection{Measurement of Dechloranes in food and feed samples}

3.4.1. Analytical parameters for quantitation and limits of quantitation Measurements of all targeted Dechloranes were performed by isotopic dilution (ID) using commercially available labeled stan- dards $\left({ }^{13} \mathrm{C}_{10}\right.$-labeled Dec 602 and ${ }^{13} \mathrm{C}_{10}$-labeled DP syn). Because of similar structures and retention times, ${ }^{13} \mathrm{C}_{10}$-labeled Dec 602 was used as internal standard for Mirex, Dec 602, Dec 603 and $\mathrm{CP}$, while ${ }^{13} \mathrm{C}_{10}$-labeled DP syn was the internal standard for the quantitation of DP syn and anti isomers. For each compounds two MRM transitions were monitored, one for quantitation and one for confirmation (usually the quantitation transition with a +2 Da offset) to ensure the adequate specificity. In practice, criteria 
used to ensure proper identification, calculations, and determination of limits of quantitation (LOQs) were the same than the ones listed in the stringent EU legislation for measurements of dioxins in food and feed (Commission Regulation EU 589/2014; Commission Regulation EU 709/2014). The calibration range (Supplementary Information, Table S1) varied for each compound, based on its own dynamic range, reproducibility at low and high level, and average response factor deviation.

Method limits of quantitation (mLOQs) varied with sample matrices because of the different sample preparation procedures and sample intake. Based on the EU legislation for dioxins, these mLOQs were assessed either based on blank levels, or from the instrumental limits of quantitation (iLOQs) when no signal was recorded from blanks. In any cases, $\mathrm{MLOQ}$ values were kept inside the calibration range. iLOQ values were assessed by means of 8 replicate injections of the lowest calibration point (iLOQ $=10 *$ standard deviation associated to the replicates), and $\mathrm{mLOQ}$ values were assessed using procedural blanks ( $\mathrm{mLOQ}=$ average blank value $+6 *$ standard deviation associated to the population $(n=7))$. When no signal was recorded for Dechloranes in the blank, and when iLOQs were lower than the first calibration point, mLOQs were determined from the first calibration point, corrected by the sample intake. Table S3 (Supplementary Information) shows all mLOQs calculated for all compounds and matrices. Values are reported in pg/g fat for most matrices, except for salmon, feed additives, and corn, for the sake of harmonization with the EU legislation for dioxins.

\subsubsection{Food and feed matrices}

Different food and feed matrices were analyzed in order to assess average daily intake for human based on food habits of the Belgian population. A total of 88 samples from 9 different food and feed matrices, as well as 16 blank samples were prepared following specific sample procedures. Six Dechlorane concentrations were measured but Dec 604 was not quantitated due to the very low response at the level of interest. Results were calculated according to the method of reporting used for dioxin analysis under the EU Legislation in terms of lower-bound (lb) and upper-bound (ub). The first approach consists in reporting zero for the target whenever the level measured in the sample is below the $\mathrm{mLOQ}$, hence an underestimated result. The upper-bound approach overestimates the final result and consists in reporting mLOQ whenever the signal coming from the sample is below the $\mathrm{mLOQ}$, even though no signal at all is recorded. The mLOQ value is therefore a very important parameter and is the threshold value (determined using blanks) establishing whether or not a signal is coming statistically from the sample and not from the background. Because rather low Dechlorane levels were observed in this study (Table 1), we reported both lower-bound and upper-bound values in Fig. 4.

Because the analytical method also allows to consider the measurement of dioxin, their TEQ levels are also reported in Table 1 to demonstrate that selected samples were not particularly contaminated from dioxins and could be considered as representative of what is available on the Belgian food market. Fig. 4 represents upper-bound and lower-bound value as respectively the top and bottom of the box. Error bars represent $\mathrm{ub}+2 *$ standard deviation. The 'true' concentrations are therefore inside the interval defined by the box. A good indication of each matrix background level is given by the position of the bottom of the box: the closer the bottom to zero, the lower is the average level as we reported zero when measured signals were below mLOQ (see chicken and corn). In all samples, levels of DP were lower than already reported in Korea (Kim et al., 2014) and in the same order than reported in foodstuffs from Japan (Kakimoto et al., 2014), even though we used a different approach with the upper- and lower-bound results.

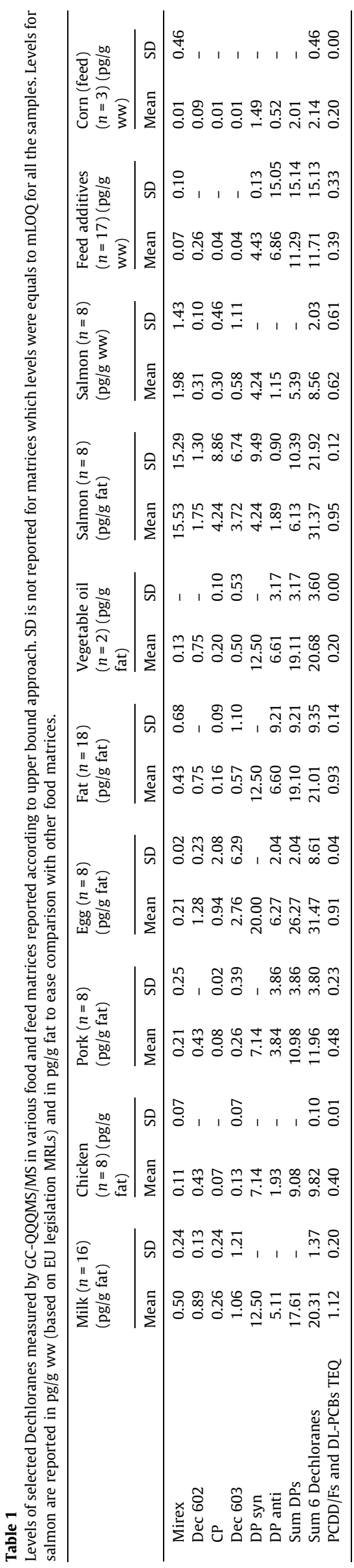




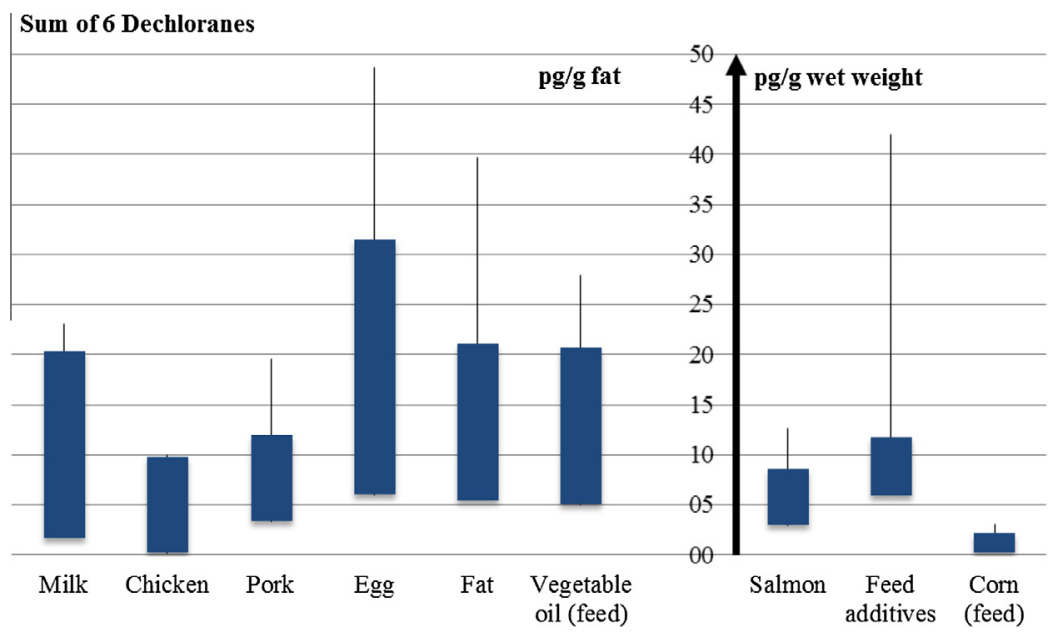

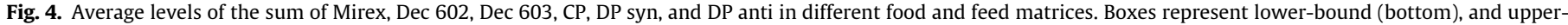

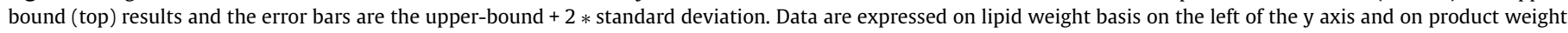
basis on the right. Salmon values are given in wet weight basis following the European Regulation for dioxins (Commission Regulation EU 1881/2006).

DP isomers were the major contributors for all matrices with the fractional abundance of the DP anti isomer $\left(f_{\text {anti }}\right.$, calculated by dividing the concentration of DP anti by the sum of the concentrations of both DP isomers) equals to $0.3 \pm 0.05$, except for feed additives, which was not in accordance with the composition of the DP technical solution. This result was however due to the fact that mLOQ for DP syn was $50 \mathrm{pg} / \mathrm{g}$, higher than DP anti, and most of the time we measured levels below $\mathrm{MLOQ}$, hence an apparent higher level in DP syn. We report the contribution of the 6 Dechloranes for all matrices in upper-bound results (Supplementary Information, Fig. S3) and in lower-bound results in Fig. 5 that only displays Dechloranes that were actually quantitated in samples.

Two out of the 17 feed additive samples exhibited high levels of DP anti (43.9 pg/g and $49.6 \mathrm{pg} / \mathrm{g}$ ), while no DP syn was measured at all. We were not able to explain these high levels, resulting in high standard deviation for this matrix. Nevertheless, this is possibly a clue to identify unexpected contamination and will have to be confirmed by a large-scale Belgian national study in all kind of foods. In salmon, we measured relatively high levels of Mirex, found in all samples $(n=8)$ above $\mathrm{mLOQ}$, in comparison with the other matrices and other Dechloranes. Mirex was especially found in fatty
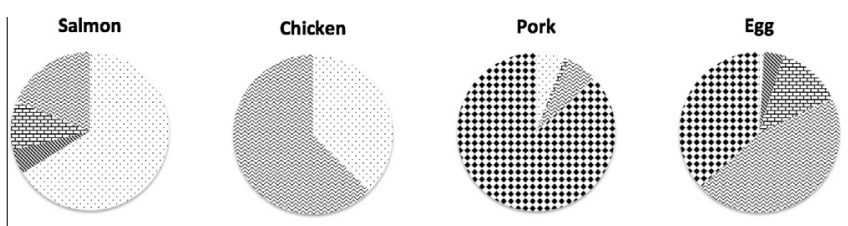

Fat
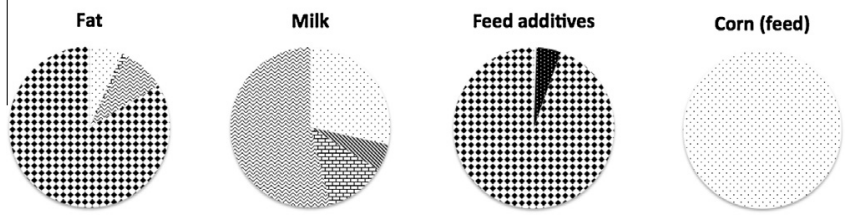

Vegetable oil (feed)
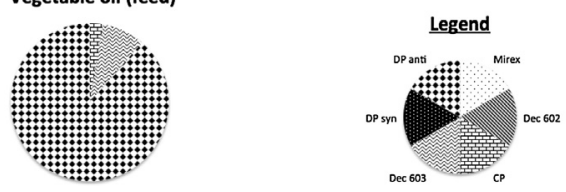

Fig. 5. Relative contribution of the 6 Dechloranes to the sum for each investigated matrix, based on lower-bound results. samples like in various pure animal fat (50\% of samples), in pork ( $75 \%$ of samples), and in milk ( $87 \%$ of samples). For corn, we only measured Mirex in a few samples, resulting in a single component pie chart, but at very low levels as we see in Fig. 4.

\subsection{Estimation of Dechlorane dietary intake}

The levels of Dechloranes that we measured in food were used to estimate an average daily intake for the Belgian population and produce a first time point for further studies. We based the calculation on the overestimated result (upper-bound) to produce a worst-case scenario. Results of these calculations are presented in Table 2 and were performed according to food habits of the Belgian population following the last food survey in Belgium (De Vriese et al., 2005) and Belgian food composition tables (Nubel, 2010).

The selected matrices were not accounting for all food consumption but were selected to represent commonly consumed goods, which allowed giving preliminary data on selected items in order to appreciate if food consumption could be a significant route of human exposure to Dechloranes. Despite the fact that levels appeared to be lower than those measured in food from Korea (Kim et al., 2014), the reported levels however suggest that Dechloranes enter the food chain and that humans are exposed via consumption of food. Based on levels we previously reported in human blood, more extensive and larger studies should be carried out to better understand fates of Dechloranes and estimate the relative contribution of food consumption to global intake. From what we have seen, at this stage, no class of compounds can be high-

Table 2

Estimated average dietary intake for the sum of 6 Dechloranes (Mirex, Dec 602, Dec 603, CP, DP syn, DP anti) measured in selected food matrices in Belgium.

\begin{tabular}{|c|c|c|c|c|c|}
\hline & \multicolumn{3}{|c|}{$\begin{array}{l}\text { Estimated dietary } \\
\text { consumption }\end{array}$} & \multirow{2}{*}{$\begin{array}{l}\text { Sum } 6 \text { Dechloranes } \\
\text { (see Table } 1 \text { ) } \\
\text { pg/g fat or } \mathrm{pg} / \mathrm{g} \text { ww }\end{array}$} & \multirow{2}{*}{$\begin{array}{l}\text { Dechloranes } \\
\text { sum intake } \\
\text { pg/day }\end{array}$} \\
\hline & g/day & $\begin{array}{l}\mathrm{g} \text { fat/ } \\
100 \mathrm{~g}\end{array}$ & $\begin{array}{l}\mathrm{g} \\
\text { fat/day }\end{array}$ & & \\
\hline Salmon & 2.7 & 16.5 & 0.4 & 8.6 & 23.1 \\
\hline Chicken & 18.4 & 9.3 & 1.7 & 9.8 & 16.8 \\
\hline Pork & 30.2 & 9.2 & 2.8 & 12.0 & 33.2 \\
\hline Egg & 9.6 & 11.3 & 1.1 & 31.5 & 34.1 \\
\hline Milk & 89.1 & 1.6 & 1.4 & 20.3 & 29.0 \\
\hline \multicolumn{5}{|c|}{ Estimated Dechloranes dietary intake (pg/day) } & 136.2 \\
\hline
\end{tabular}


lighted as strongly contributing to human exposure to Dechloranes. These results will further be cross-compared with other possible routes of exposure via measurements in other matrices such as dust.

\section{Conclusions}

A dedicated analytical method has been developed for the measurement of six members of the Dechlorane family (Mirex, Dec 602 , Dec 603, CP, DP syn, DP anti) in food and feed. The use of PTV and GC-IDQQQMS/MS permitted to perform measurements at the low pg level, ensuring detection of all targets, except Dec 604. The optimization steps focused on the improvement of the sensitivity from the clean-up of samples to their injection and detection. We subsequently analyzed 88 samples from 9 different matrices, representing commonly consumed goods, in order to preliminary assess a daily intake of Dechloranes from these products. Levels in food were found to be close to the method limits of quantitation, except for some samples, whose contamination appeared to be significantly higher. An estimation of the dietary intake showed that this was probably not the single route of exposure. Further studies are necessary to understand how food samples are getting contaminated by Dechloranes and whether or not these levels could be harmful to human. Reported levels will also be integrated in a global study considering dust and other possible routes of exposure to conclude with regards to the significance of food as route of human exposure to Dechloranes.

\section{Acknowledgements}

The Research Training Fund for Industry and Agriculture (F.R.I.A) is thanked for providing a Ph.D. bursary to B. L'Homme. C. Calaprice was financially supported partly by Fondo Giovani for PhD students at the Polytechnic University of Bari, and partly from the Inter-polytechnic School (Milan, Turin, Bari). Thanks to Fluid Management Systems Inc. for providing sample clean-up consumables for this study.

\section{Appendix A. Supplementary material}

Supplementary data associated with this article can be found, in the online version, at http://dx.doi.org/10.1016/j.chemosphere. 2015.07.043.

\section{References}

Brasseur, C., Pirard, C., L'Homme, B., Focant, J.-F., 2012. Measurement of human levels of dechlorane 602 by CZC-NCI-HRTOFMS. Organohalogen Compd. 74, 5558.

Brasseur, C., Pirard, C., Scholl, G., De Pauw, E., Viel, J.F., Shend, L., Reiner, E.J., Focant J.F., 2014. Levels of dechloranes and polybrominated diphenyl ethers (PBDEs) in human serum from France. Environ. Int. 65, 33-40.

Cao, Z., Xu, F., Covaci, A., Wu, M., Wang, H., Yu, G., Wang, B., Deng, S., Huang, J., Wang, X., 2014. Distribution patterns of brominated, chlorinated, and phosphorus flame retardants with particle size in indoor and outdoor dust and implications for human exposure. Environ. Sci. Technol. 48, 8839-8846.

Cequier, E., Marcé, R.M., Becher, G., Thomsen, C., 2013. Determination of emerging halogenated flame retardants and polybrominated diphenyl ethers in serum by gas chromatography mass spectrometry. J. Chromatogr. A 1310, 126-132.
Commission Regulation (EC) 1881/2006 of 19 December 2006 setting maximum levels for certain contaminants in foodstuffs, 2006. Off. J. Eur. Commun. L364, $5-24$.

Commission Regulation (EU) No 589/2014 of 2 June 2014 laying down methods of sampling and analysis for the control of levels of dioxins, dioxin-like PCBs and non-dioxin-like PCBs in certain foodstuffs and repealing Regulation (EU) No 252/2012, 2014. Off. J. Eur. Commun. L164, 18-40.

Commission Regulation (EU) No 709/2014 of 20 June 2014 amending Regulation (EC) No 152/2009 as regards the determination of the levels of dioxins and polychlorinated biphenyls, 2014. Off. J. Eur. Commun. L188, 1-18.

De Vriese, S., Debacker, G., De Henauw, S., Huybrechts, I., Kornitzer, M., Leveque, A., Moreau, M., Van Oyen, H., 2005. The Belgian food consumption survey: aims, design and methods. Arch. Public Health 63, 1-16.

Dodson, R.E., Perovich, L.J., Covaci, A., Van den Eede, N., Ionas, A.C., Dirtu, A.C., Green Brody, J., Rudel, R.A., 2012. After the PBDE phase-out: a broad suite of flame retardants in repeat house dust samples from California. Environ. Sci. Technol. 46, 13056-13066.

Focant, J.-F., Eppe, G., Pirard, C., De Pauw, E., 2001. Fast clean-up for polychlorinated dibenzo-p-dioxins, dibenzofurans and coplanar polychlorinated biphenyls analysis of high-fat-content biological samples. J. Chromatogr. A 925, 207-221.

Focant, J.-F., Pirard, C., De Pauw, E., 2004. Automated sample preparationfractionation for the measurement of dioxins and related compounds in biological matrices: a review. Talanta 63, 1101-1113.

Focant, J.-F., Eppe, G., Massart, A.-C., Scholl, G., Pirard, C., De Pauw, E., 2006. Highthroughput biomonitoring of dioxins and polychlorinated biphenyls at the subpicogram level in human serum. J. Chromatogr. A 1130, 97-107.

INCHEM, 1984. International Programme on Chemicals safety, Environmental Health Criteria 44, United Nations Environment Programm. <http://www. inchem.org/documents/ehc/ehc/ehc44.htm> (accessed March 2015).

Jobst, K.J., Shen, L., Reiner, E.J., Taguchi, V.Y., Helm, P.A., McCrindle, Rd., Backus, S., 2013. The use of mass defect plots for the identification of (novel) halogenated contaminants in the environment. Anal. Bioanal. Chem. 405, 3289-3297.

Kakimoto, K., Nagayoshi, H., Takagi, S., Akutsu, K., Konishi, Y., Kajimura, K., Hayakawa, K., Toriba, A., 2014. Inhalation and dietary exposure to Dechlorane Plus and polybrominated diphenyl ethers in Osaka, Japan. Ecotoxicol. Environ. Saf. 99, 69-73.

Kim, J., Sona, M.H., Kim, J., Suh, J., Kang, Y., Chang, Y.S., 2014. Assessment of Dechlorane compounds in foodstuffs obtained from retail markets and estimates of dietary intake in Korean population. J. Hazard. Mater. 275, 19-25.

L'Homme, B., Scholl, G. Eppe, G., Focant, J.F., 2015. Validation of a gas chromatography-triple quadrupole mass spectrometry method for confirmatory analysis of dioxins and dioxin-like polychlorobiphenyls in feed following new EU Regulation 709/2014. J. Chromatogr. A 1376, 149-158.

Mac Namara, K., Leardi, R., Sabuneti, A., 2005. Fast GC analysis of major volatile compounds in distilled alcoholic beverages: optimisation of injection and chromatographic conditions. Anal. Chim. Acta 542, 260-267.

Muñoz-Arnanz, J., Roscales, J.L., Vicente, A., Aguirre, J.I., Jiménez, B., 2012. Dechlorane plus in eggs of two gull species (Larus michahellis and Larus audouinii) from the southwestern Mediterranean Sea. Anal. Bioanal. Chem. 404, $2765-2773$.

NUBEL, 2010. Belgian Food Composition Table, fourth ed. Ministry of Public Health, Brussels, Belgium.

OxyChem, 2007. Dechlorane Plus Manual, Ver: 7-27-07. <http://www.oxy.com/ OurBusinesses/Chemicals/Products/Documents/dechloraneplus/ dechlorane_plus.pdf> (accessed March 2015).

Pakalin, S., Cole, T., Steinkellner, J., Nicolas, R., Tissier, C., Munn, S., Eisenreich, S., 2007. Review on Production Processes of Decabromodiphenyl Ether (DECABDE) Used in Polymeric Applications in Electrical and Electronic Equipment, and Assessment of the Availability of Potential Alternative to DECABDE. Institute of Health and Consumer Protection, Joint Research Centre, European Commission. pp. 9-11.

Pirard, C., Focant, J.-F., De Pauw, E., 2002. An improved clean-up strategy for simultaneous analysis of polychlorinated dibenzo-p-dioxins (PCDD), polychlorinated dibenzofurans (PCDF), and polychlorinated biphenyls (PCB) in fatty food samples. Fresen. J. Anal. Chem. 372, 373-381.

Shen, L., Jobst, K.J., Helm, P.A., Reiner, E.J., McCrindle, R., Tomy, G.T., Backus, S., Brindle, I.D., Marvin, C.H., 2012. Identification and determination of the dechlorination products of Dechlorane 602 in Great Lakes fish and Arctic beluga whales by gas chromatography-high resolution mass spectrometry. Anal. Bioanal. Chem. 404, 2737-2748.

Zhou, S.N., Siddique, S., Lavoie, L., Takser, L., Abdelouahab, N., Zhu, J., 2014. Hexachloronorbornene-based flame retardants in humans: levels in maternal serum and milk. Environ. Int. 66, 11-17. 